\title{
Sustainable Entangled State of Two Qutrits Under Laser Irradiation
}

\author{
A.A. Biryukov and M.A. Shleenkov ${ }^{\text {a }}$ \\ Samara State University, 1 Akademik Pavlov Str. Samara 443011, Russia
}

\begin{abstract}
We study the evolution of quantum entanglement in the model of two identical qubits interacting with a single-mode laser field. The density matrix and Peres-Horodecki parameter are calculated within the frameworks of path-integral formalism. The quantum entanglement measure is shown to be strongly dependent upon the phase difference between the laser radiation acting on each cubit. This observation may offer the possibility of quantum entanglement stationary control by varying the distance between the qubits.
\end{abstract}

Quantum entanglement has been a subject of intense theoretical and experimental studies during the last decades because of its significance in understanding quantum mechanics and promising applications. Fundamental principles and basic methods of creating and detecting entangled states in different quantum systems can be found, e.g., in the review [1]. Multiple potential applications of quantum entanglement phenomena include quantum computer [2], quantum cryptography [3], quantum teleportation [4], etc.

A few dimensionless parameters were introduced to measure quantum entanglement quantitatively, e.g., the Peres-Horodecki criterion [5], the concurrence [6], the negativity [7], etc.

In the present paper we consider two qubits interacting with a mode of laser field. We develop a mathematical method (based on path integral approach) for calculating the density matrix and the Peres-Horodecki parameter and analyze the quantum entanglement dynamics depending on variable experimental parameters: intensity and phase difference of laser fields acting on the cubits.

In this approach we study the possibility of high entanglement qubits states creation and containment.

\section{References}

1. R. Horodecki, P. Horodecki, M. Horodecki, et al, Rev. Mod. Phys. 81, 865-942 (2009)

2. R. Raussendorf and H. J. Briegel, Phys. Rev. Lett. 86, 5188-5191 (2001)

3. W. Klobus, A. Grudka, Baumgartner, et al Phys. Rev. B 89, 125404-1 (2014)

4. J. M. Torres, J. Z. Bernad, and G. Alber,. Phys. Rev. A 90, 012304 (2014)

5. A. Peres, Phys. Rev. Lett. 77, 1413-1415 (1996)

6. W. K. Wootters, Phys. Rev. Lett. 80, 2245-2248 (1998)

7. G. Vidal, R. F. Werner, Phys. Rev. A 65, 032314 (2002)

\footnotetext{
${ }^{\text {a }}$ Corresponding author: shleenkov@list.ru
} 